\title{
Factors influencing adolescent whole grain intake: In-depth interviews with adolescents using SenseCam technology
}

\author{
M. Kamar ${ }^{1}$, C.E.L. Evans ${ }^{1}$ and S. Hugh-Jones ${ }^{2}$ \\ ${ }^{1}$ Nutritional Epidemiology Group, School of Food Science and Nutrition, University of Leeds, LS2 $9 J T$ and ${ }^{2}$ School of \\ Psychology, Faculty of Medicine and Health, University of Leeds, LS2 9JT
}

Whole grain consumption is associated with reduced risks of chronic disease ${ }^{1}$. One-fifth of UK adults and children do not consume whole grain, and adolescents have particularly low consumption rates ${ }^{2}$. There is little research on correlates of whole grain consumption in this age group. This study aimed to explore the socio-economic, environmental, lifestyle, and psychological factors influencing adolescent whole grain intake via in-depth interviews, using SenseCam Technology ${ }^{3}$.

Eight participants ( 4 males; 4 females) from the city of Leeds, UK, took part in the study (aged 11-16 years). Participants wore SenseCam for three days, where it auto-captured photos every twenty seconds. Then participants undertook traditional 24-hour recalls and in-depth interviews for attitudes, knowledge and consumption of wholegrain foods; as well as barriers and facilitators to consumption. SenseCam images prompted conversation during the interviews, which were audio-recorded and analysed using inductive content analysis.

The majority of participants had tried wholegrain foods in the past. Adolescents were more influenced by parents and online media than by peers. Most adolescents related "whole grains" to wholemeal toast, and were not aware that varieties they already consumed, such as popcorn, quinoa and brown rice, were whole grain as well. Barriers to consumption included difficulties in identifying them and their health benefits, taste and visual appeal as well as poor availability outside the home. Suggested facilitators to consumption were advertisements, awareness campaigns, improved sensory appeal, increased availability and variety, and tailoring of products for young people. SenseCam use was helpful in prompting open, useful conversation as well as highlighting issues with self-reported dietary recall and whole grain identification. This study is a formative stage in building of questionnaires that explore correlates and quantifies whole grain intake in a representative group of UK adolescents. Outcomes of this qualitative study may inform research and health promotion, to increase whole grain intake in this age group.

1. McKeown NM, Jacques PF, Seal CJ et al. (2013) $J$ Nutr 143, 744S-758S

2. Mann KD, Pearce MS, McKevith B et al. (2015) Br J Nutr 113, 1643-1651.

3. Gemming L, Rush E, Maddison R et al. (2015) Br J Nutr 113, 284-91., 113, 284-291. 